\title{
Adaptação do Rich Picture (Desenho Rico) no Processo de Análise para o Desenvolvimento de Softwares no Curso Técnico em Informática
}

\author{
Adaptation of Rich Picture for Analysis Process \\ In Software Development on Technical Course of Computing
}

\begin{abstract}
Resumo: Este estudo objetiva mostrar o uso do método do desenho rico como parte do processo de desenvolvimento de Trabalho de Conclusão de Curso em um programa de ensino técnico profissionalizante. Para compreender a relevância do uso da técnica do desenho rico, utilizou-se como dados a opinião de 108 estudantes. Foram elencadas as informações obtidas no decorrer dos anos de 2010 e 2011 com todos os estudantes de último ano do curso em uma unidade do Serviço Nacional de Aprendizagem Industrial (SENAI). O instrumento de coleta de informações foi a entrevista aberta e a análise foi baseada no método análise de conteúdo. Como resultado, a ferramenta de desenho rico contribuiu não só para o processo de ensino-aprendizagem, como também, para entendimento de uma situação-problema na análise de sistemas.
\end{abstract}

Palavras-chave: Desenho rico. Desenvolvimento de softwares. Ensino.

Abstract: This study aims to show the use of the rich picture as a auxiliary method to elaborate a conclusion work on technical course of computing. To understand the relevance of the technique of rich picture, the opinion of 108 students was collected. The opinions has been collected during the years 2010 and 2011 with students of final year in a unit of the Serviço Nacional de Aprendizagem Industrial (SENAI). The instrument for data collection was the open interviews and the analysis was based on content analysis method. As a result, the tool of rich picture not only contributed to the process of teaching and learning, but also for understanding of a problem situation in systems analysis.

Keywords: Rich Picture. Software Development. Teaching Techniques.

ADÃO, Nilton Manoel Lacerda; MELO, Alexandre Altair de; GRUMOVSKI Dieison. Adaptação do rich picture (desenho rico) no processo de análise para o desenvolvimento de softwares no curso Técnico em Informática. Informática na Educação: teoria e prática, Porto Alegre, v. 17, n. 1, p. 3543, jan./jun. 2014.

\section{Nilton Manoel Lacerda Adão}

Universidade Federal de Santa Catarina

Alexandre Altair de Melo
Pontifícia Universidade Católica do Paraná

Dieison Grumovski

Centro Universitário Internacional

\section{Introdução}

P ensar para agir sistemicamente pode ser considerado um método de investigação e ação para definir estratégias em diferentes áreas do conhecimento. Partindo dessa possibilidade de compreensão será tratado neste artigo como abordagem sistêmica, todo e qualquer esforço para se adotar uma epistemologia, uma teoria ou uma prática sistêmica (SCHLINDWEIN, 2004).

A forma sistêmica de compreender o mundo define as abordagens "hard" e "soft" para a compreensão da sistemicidade (BERNARDO et al., 2008). A abordagem hard systems con- 
siste na visão de mundo sistêmico em que os sistemas são perceptíveis pelo observador. Já a abordagem soft systems consiste no processo de indagação sistêmica (CHECKLAND apud BERNARDO et al., 2008). Ou seja, na abordagem soft o observador lida com situações de complexidade para organizar e delimitar o seu sistema de interesse considerando a interpretação de quem está envolvido sobre a situação em que se insere, em fluxo de informações e sistema de aprendizagem contínuo. A figura a seguir representa a diferença entre abordagem soft e hard.

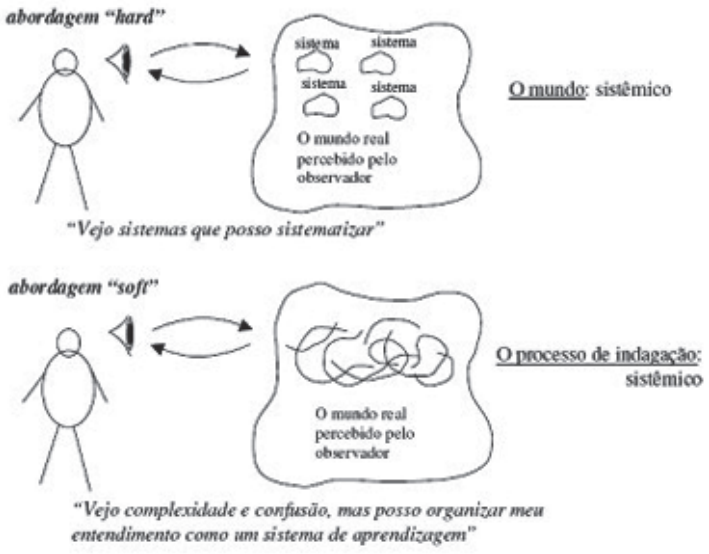

Figura 1. Representação das abordagens hard e soft systems

Fonte: Sshlindwein (2004)

Partindo da relação de complexidade existente, este estudo delimita-se na abordagem soft. O Desenho Rico é uma das etapas da Soft Systems Methodology (SSM) originada nos anos de 1960 com o trabalho por Peter Checkland e seus alunos em Lancaster University (MONK; HOWARD, 1998). No SSM o desenho rico faz parte de um processo inicial que consiste em entender uma situação-problema para compreender a realidade na qual uma situação de trabalho ou atividades podem ser estabe- lecidas como um sistema. A figura 2 mostra o processo da SSM.

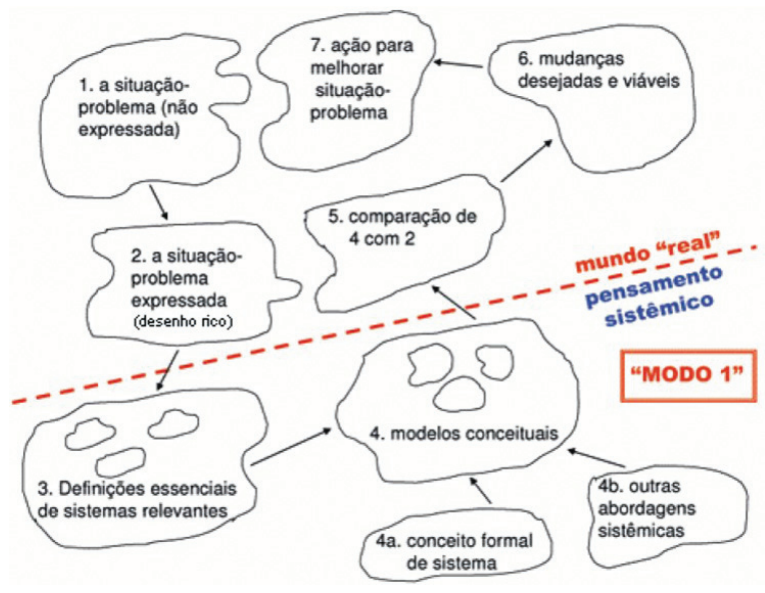

Figura 2. Processo da SSM Fonte: Bernardo, et. al. (2008)

Este artigo limita-se nos dois processos iniciais que resultam na aplicação do Desenho Rico adaptado ao processo de ensino aprendizagem de um Curso Técnico em informática para o desenvolvimento de softwares. De acordo com Monk e Howard (1998), o método de desenvolvimento do desenho rico inicia-se com entrevistas que de forma ideal deve ser realizada no local de trabalho do entrevistado para que seja possível conhecer documentos, produtos e até mesmo observar as ações desenvolvidas. Segundo estes autores, será possível organizar as informações fornecidas que depois devem ser representadas em desenho para posteriormente serem validadas com os envolvidos de maneira que o resultado final seja uma representação gráfica proveniente de um processo interativo de entendimento e refino para se chegar à compreensão (MONK; HOWARD, 1998). Na figura 3 apresenta-se a aplicação de um Desenho Rico, mediante uma situação-problema. 


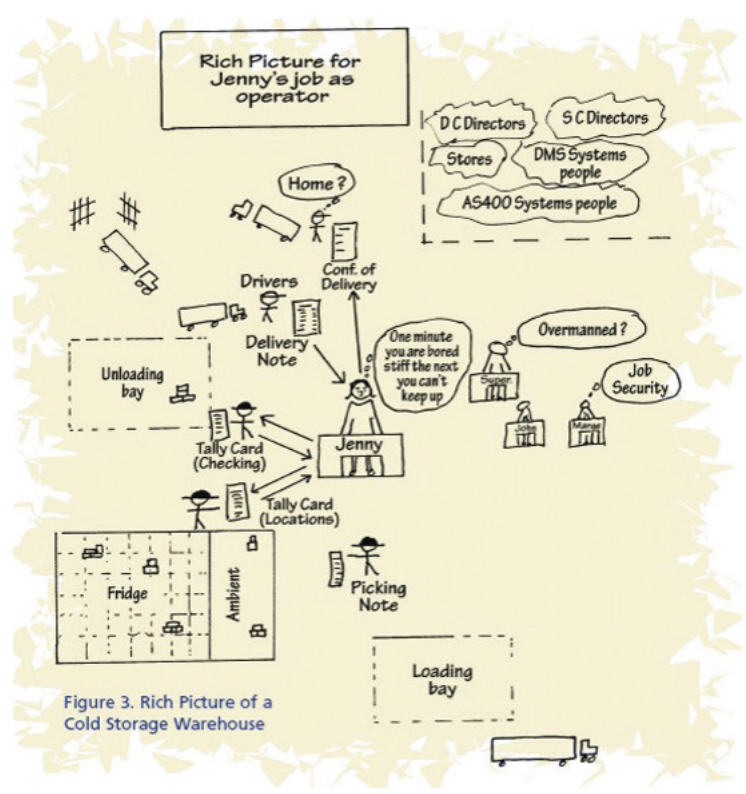

Figura 3. Exemplo de Desenho Rico Fonte: Monk e Howard (1998).

Observa-se que o desenho (fig. 3) representa fatores físicos e não-físicos de um sistema de relações. Vicente (2005) destaca que, ao abordar uma visão da tecnologia com a inclusão dos aspectos físicos e não-físicos no desenho de um sistema, é possível ao incorporar o fator humano, melhorar os ambientes de trabalho e as atividades humanas.

O desenvolvimento de um software permite melhorar uma realidade humana otimizando processos e rotinas de trabalho. Diante disso, parte-se do principio que o conhecimento da realidade estudada possibilita que a solução proposta atenda aos interesses. Partindo desse pressuposto a técnica apresentada neste estudo para auxiliar a comunicação com terceiros, é uma adaptação de desenho rico (rich picture) para o desenvolvimento de softwares a partir da compreensão de uma situação-problema. Essa ferramenta consiste em utilizar imagens, setas, palavras e outros ícones gráficos para demonstrar de uma forma direta, o mapea- mento de um processo. Esta técnica substitui textos para dar significado a um contexto em específico.

Esse ferramental será objeto de estudo no referente artigo quando da sua aplicabilidade por estudantes de um Curso Técnico em Informática na Unidade SENAI de Joinville (SC). Para tanto, será apresentada a metodologia contextualizando o grupo estudado e a forma como foram obtidas as informações analisadas. Posteriormente, os resultados das entrevistas, serão apresentados e discutidos para mostrar a contribuição do desenho rico nas práticas de 108 estudantes, mas, que pode ser utilizada em diferentes realidades.

\section{Metodologia}

\subsection{Campo da investigação}

Foram entrevistados os estudantes de quatro turmas de um programa de Curso Técnico em Informática envolvidas no processo de desenvolvimento de Trabalho de Conclusão de Curso no ano de 2010 e 2011. Esse grupo de alunos era composto por jovens de ambos os sexos com faixa etária entre 15 e 19 anos. O foco do curso é o desenvolvimento de software pautado na capacidade de compreender uma realidade para agilizar e organizar atividades. As atividades são realizadas em equipes de até quatro estudantes que desenvolvem um software para atender às necessidades de um cliente real ${ }^{1}$. A adaptação do desenho rico

\footnotetext{
${ }^{1}$ No processo de desenvolvimento de TCCs do referido curso é necessário a existência do "cliente" para que o aluno desenvolva sua pesquisa aproximando-se das realidades e necessidades do mercado. Entretanto, é valido relatar que não existe relação comercial, os estudantes desenvolvem os softwares de forma voluntária responsabilizando-se em entregar o produto final (software).
} 
como técnica está associada à fase de entendimento da regra de negócios e do levantamento de requisitos funcionais que se constitui na fase inicial do projeto do software.

\subsection{Amostragem}

No total 108 estudantes participaram da pesquisa representando $100 \%$ dos grupos que aplicaram a técnica adaptada do desenho rico no desenvolvimento de TCCs no Curso Técnico em Informática do SENAI em Joinville nos anos de 2010 e 2011.

\subsection{Aplicação da entrevista}

O método de coleta de dados aplicado foi a entrevista aberta. Compreende-se por entrevista aberta aquela em que o entrevistador apresenta uma questão ou tema inicial e o entrevistado constrói seus argumentos seguindo suas preferências sem intervenção do entrevistador (MARTINS, 2001). O foco da pesquisa foi compreender como os estudantes qualificavam o uso da técnica do desenho rico na fase de pré-análise que consiste na compreensão de uma situação-problema para o desenvolvimento de softwares.

Os estudantes foram divididos em grupos de 4 integrantes para possibilitar a discussão sobre o uso do desenho rico e para chegar ao consenso sobre a viabilidade, ou não, do uso do desenho rico no processo de desenvolvimento de software. Para atingir este resultado, a questão que permeou a entrevista foi: opine sobre o uso do desenho no seu desenvolvimento de Trabalho de Conclusão de Curso?

\subsection{Análise do resultado}

Para análise das informações coletadas empregou-se Análise de Conteúdos fomentada por Bardin (2009). Destarte, inicialmente os dados das entrevistas foram divididos em duas categorias: 1) desenho rico é pertinente, ou, 2) não é pertinente no desenvolvimento de software. Nesta primeira classificação, observou-se que $100 \%$ dos entrevistados consideraram que a técnica adaptada do desenho rico foi pertinente para o entendimento de uma situação-problema.

A partir deste resultado, foram elencadas as falas com maior representatividade no que se refere à percepção dos entrevistados. Ao mesmo tempo, houve preocupação dos autores em apresentar os dados que representam a opinião somente do grupo entrevistado. Somente após apresentação dos dados, é que as discussões representam a opinião dos autores. Para ilustrar as informações alguns desenhos desenvolvidos pelo grupo estudado também serão apresentados.

\section{Resultados}

\subsection{Uso do desenho rico adaptado}

A educação profissional, segundo a Lei de Diretrizes e Bases da Educação - LDB 9394/96 - é concebida como "integrada às diferentes formas de educação, ao trabalho, à ciência e à tecnologia, conduzindo ao permanente desenvolvimento de aptidões para a vida produtiva" (Art. 39 - LDB), constituindo-se em uma importante estratégia para que os cidadãos tenham efetivo acesso às conquistas científicas e tecnológicas da sociedade. 
Seguindo esse principio régio da LDB, os cursos técnicos independente da área de formação prezam a formação dos estudantes na questão do saber fazer, valendo-se de todo um ferramental de cada área do conhecimento para que ele escreva, desenvolva e crie esse saber. Contudo tendem a existir situações, principalmente num curso ligado a área de Tecnologia da Informação (TI), independente do nível de ensino, que os estudantes necessitam interagir com terceiros além do ambiente acadêmico, para sanar, por exemplo, dúvidas sobre determinado processo ou mesmo para demonstrar de uma forma não técnica a modelagem de um sistema.

Este trabalho adapta, com base na primeira etapa de desenvolvimento da SSM, o desenho rico desenvolvido pelo grupo estudado para melhorar o processo de desenvolvimento de softwares. O método do desenho rico, neste caso, objetiva representar uma situação-problema considerando, estrutura, pessoas envolvidas, papéis exercidos e a relação com os organismos externos. Na figura 3 observa-se as interações entre as atividades internas de uma Entidade Filantrópica que atende deficientes visuais e de baixa visão e a importância que deve ser dada aos documentos e dados que contribuem para um sistema de informações entre a instituição e entidades externas para obtenção de recursos financeiros, aumento da empregabilidade e integração social.

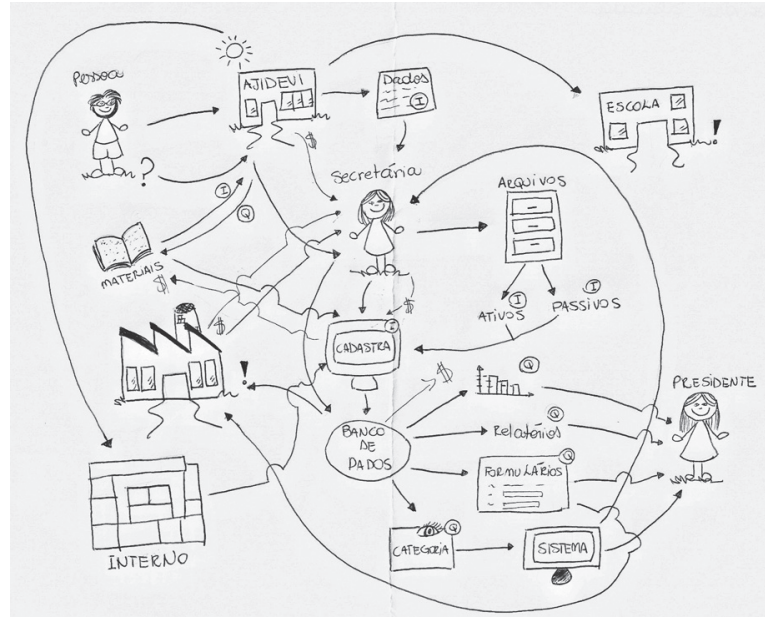

Figura 3. Desenho Rico com foco em documentação Fonte: TCC de um grupo entrevistado (2011).

Obs. : o título foi retirado para não expor a instituição representada pelo desenho rico.

A partir do momento que os estudantes passam a ter compreensão da realidade em que irão interagir, a possibilidade de propor uma solução baseada em software se mostra mais clara, e conforme Pressman (2011) compreender o problema (comunicação e análise) auxilia na busca dessa solução. Nesse sentido de compreensão do problema e segundo Monk e Howard (1998) no desenho rico estão contidos pontos de vistas, interesses, fluxos de informações, mercadorias, dados e documentos.

Para exemplificar as especificidades de cada situação-problema também será apresentada a figura 4 que representa um sistema de negócios onde se trabalha por encomenda de produtos. Observa-se neste caso, a interação entre as informações requeridas por um cliente e o setor produtivo. Um detalhe pertinente sobre este desenho rico consiste na importância dada ao banco que faz parte do sistema onde os clientes realizam o pagamento que consiste no inicio das operações de produção e contábeis. 


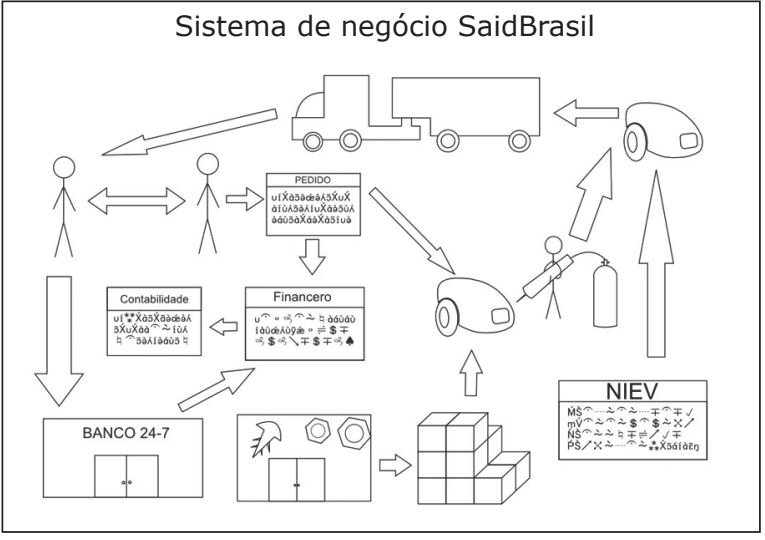

Figura 4. Desenho Rico com foco em produção Fonte: TCC de um grupo entrevistado (2010). Obs.: o título foi retirado para não expor a instituição representada pelo desenho rico.

\subsection{Percepções sobre a aplicação do desenho rico}

Os depoimentos apresentados foram elencados considerando a relevância na representação da totalidade. Segundo os entrevistados, ao refletir sobre a situação-problema da realidade estudada foi possível conhecer com detalhes os problemas no processo de gerenciamento das instituições:

O Desenho Rico foi uma etapa essencial na análise do nosso sistema para que pudéssemos ter visualizado todo o funcionamento do escritório, ilustrativamente, identificando com detalhes as partes falhas do gerenciamento atual do nosso cliente (Grupo de entrevistados 1).

Destaca-se a importância desta compreensão para propor software que atendam às necessidades dos clientes:
O Desenho Rico é uma forma mais fácil de ver a aplicabilidade do sistema². Ele auxiliará para que se entenda o que deve ser feito, como deve ser feito bem como as funções e processos que conterão o sistema (Grupo de entrevistados 2).

Outra questão considerada pertinente, foi a percepção dos clientes quanto a apresentação da descrição da realidade na qual estão envolvidos. Por meio da representação do Desenho Rico, os clientes se compreenderam como parte de um processo expressando a realidade na qual estavam inseridos:

A elaboração do Desenho Rico foi de muita importância para o processo de construção do sistema, sendo que com ele o entendimento do processo sugerido pela cliente foi de forma mais clara e bem definida (Grupo de entrevistados 3 ).

Ao reconhecer a pertinência do método, somente depois que a situação-problema do cliente foi "clarificada" na forma de desenho rico é que o processo de desenvolvimento de software foi continuado:

O Desenho Rico foi importante para explanar e esclarecer as informações trazidas do primeiro encontro auxiliando posteriormente no Relatório de Intenções e nos requisitos funcionais (Grupo de entrevistados 4).

Como resultado final, o uso do desenho rico permitiu aos estudantes interpretar, interagir e agir diante de uma situação-problema destacando e propondo melhorias de cada processo mediante desenvolvimento de um software:

\footnotetext{
${ }_{2}$ Neste comentário os estudantes tratam sistema como sinônimo de software o que não é incorreto. Entretanto por uma questão metodológica e conceitual os autores não tratarão desta forma na discussão proposta.
} 
O Desenho Rico foi importante para que conseguíssemos compreender exatamente as dificuldades do nosso cliente juntamente com o processo realizado no Pet-Shop e assim, conseguiremos realizar melhorias no sistema, deixando-o mais prático e facilitando a sua utilização (Grupo de entrevistados 5).

Destaca-se que assim como a palavra "sistema" possui vários conceitos, também "modelo" oferece algumas dezenas de aplicações (AUDY, 2005). O uso do desenho rico como um modelo para se visualizar a resposta a uma situação problema auxiliou os estudantes durante o processo de ensino-aprendizagem, na busca de uma solução aos problemas que Ihes foram apresentados.

\section{Discussão}

Conforme afirma Sommerville (2007, p. 161) "[...] tornar a arquitetura do sistema explícita em um estágio inicial de desenvolvimento do sistema requer alguma análise". E ainda segundo Audy (2005, p. 22) "[...] a expressão "análise de sistemas" tem duas conotações. Uma delas em sentido amplo e que será abordado aqui, trata de uma aplicação do pensamento sistêmico na tentativa de solucionar problemas; a outra, em sentido mais restrito, está relacionada ao desenvolvimento de sistemas de informação com o uso de computadores e a sistemas específicos". Com isso o desenho rico complementou os diagramas de análise das abordagens clássicas de construção de sistemas, como o interativo e incremental ${ }^{3}$ usando notação da

\footnotetext{
${ }^{3} \mathrm{O}$ modelo de ciclo de vida incremental e iterativo foi proposto como uma resposta aos problemas encontrados no modelo em cascata. Um processo de desenvolvimento segundo essa abordagem divide o desenvolvimento de um produto de software em ciclos.
}

$\mathrm{UML}^{4}$. Abordagem essa vista durante o processo de ensino-aprendizagem em sala de aula. No processo de desenvolvimento de softwares a análise de sistemas antecede o seu desenvolvimento propriamente dito, e consiste em levantar o maior número possível de informações sobre o negócio do cliente, e o que ele espera com a implantação do sistema que será desenvolvido.

Nessa fase de análise, o desenho rico ajuda muito na troca de informações entre os profissionais da área de informática e o cliente, uma vez que o desenho não possui informações técnicas e é de fácil entendimento. Com isso a análise do sistema pode ficar mais completa e deixar mais claras as funcionalidades que se espera do software que será desenvolvido.

Ainda no processo de análise de sistemas, tem-se o momento de identificar os requisitos, sejam eles funcionais ou não. Segundo Jacobson (1999) "Requisitos são capacidades e condições às quais o sistema - e em termos mais amplos, o projeto - deve atender". Os requisitos funcionais têm como objetivo identificar as funcionalidades que o sistema deverá conter, enquanto que os não funcionais dizem respeito a recursos físicos, definições de tecnologias utilizadas para desenvolver o sistema, entre outros itens que não tenham relação com a parte funcional, de negócio, do software.

Sabendo desses conceitos, pode-se fazer uma ligação direta com o Desenho Rico, pois identificar requisitos, sejam eles funcionais ou não, pode ficar mais fácil quando todo o processo do cliente é disposto em uma representação gráfica. Com estes itens bem definidos, o restante da análise do sistema fica facilitado, pois toda a equipe de desenvolvimento terá uma visão maior e mais clara de todo o pro-

\footnotetext{
${ }^{4} \mathrm{~A}$ UML é uma linguagem visual para modelar sistemas orientados a objetos.
} 
cesso e de onde o software que será desenvolvido irá ser empregado, desenvolvendo com isso um sistema mais funcional, objetivo e que atenda as especificações desejadas.

\section{Considerações finais}

O uso do desenho rico, como ferramental de apoio, ajudou aos estudantes a complementar seu pensamento analítico, e no desenvolvimento da análise do sistema que se propuseram. Conhecendo a realidade das pessoas, fica mais fácil atender as necessidades e propor melhorias de processos e rotinas de trabalho.

Com base nas exposições dos entrevistados, percebeu-se a importância do desenho rico para um melhor entendimento de uma situação-problema. Além disso, com o desenho rico é possível contextualizar os estudantes em discussões sobre a necessidade de uma abordagem sistêmica na realidade vivenciada. Destarte, é possível formar estudantes que, diferente de uma educação tecnicista-mecanicista, sejam capazes de aliar o conhecimento técnico ao desenvolvimento de tecnologias nunca deixando de considerar o fator humano, tornando-se assim, profissionais mais capazes de agir e interferir na realidade.

É possível destacar, entretanto, que não se procurou medir a velocidade ou ganho em horas com uso do desenho rico, se comparado a outras técnicas clássicas para análise e construção de sistemas. Podendo ser esse ponto, objeto de estudo para trabalhos futuros.

\section{Referências}

AUDY, Jorge Luis Nicolas; ANDRADE, Gilberto Keller de; CIDRAL, Alexandre. Fundamentos de Sistemas de Informação. Porto Alegre: Bookman, 2005.

BARDIN, Laurence. Análise de conteúdo. Coimbra: Edições 70, 2009.

BERNARDO, Vanessa M. et al. Questões complexas na agricultura de Santa Catarina: estruturando situações-problema através da abordagem sistêmica. IV CONGRESSO BRASILEIRO DE SISTEMAS. 28 a 30 outubro de 2008. Anais... Uni-FACEF, França, São Paulo.

BERTALANFY, Ludwig Von. General System Theory. New Yourk: George Braziller, 2003.

CHIAVENATO, Idalberto. Planejamento e controle de produção. 2. ed. Barueri: Manole, 2008.

JACOBSON, I.; BOOCH, G.; RUMBAUGH, J. The Unified Software Development Proces. Reading, MA: Addison-Wesley, 1999.

MARTINS, Maria C. F. N. Humanização das relações existenciais: a formação do profissional de saúde. São Paulo: Casa do Psicólogo, 2001. 
MONK, Andrew; HOWARD; Steve Howard. The Rich Picture: A Tool for Reasoning About Work Context, 1998. Disponível em: <http://portablelearner.com/half-notes/rich-pictures/> Acesso em: 30 mar. 2010.

PRESSMAN, Roger S. Engenharia de Software uma Abordagem Profissional. 7. ed. Porto Alegre: Bookman, 2011.

SILVA, Gisele Cristina Sena da; MEDEIROS, Denise Dumke de. Metodologia de checkland aplicada à implementação da produção mais limpa em serviços. Gest. Prod., São Carlos, v. 13, n. 3, dez. 2006. Disponível em: <http://www.scielo.br/scielo.php?script=sci_arttext\&pid=S0104-530X2006000300005 \&lng $=p t \& n r m=i s o>$. Acesso em: 29 mar. 2010.

SCHLINDWEIN, S. L. Por que a analise sistêmica não pode refletir a realidade? Redes, Santa Cruz do Sul, v. 9, n. 2, p. 117-132, 2004.

SOMMERVILLE, Ian. Engenharia de Software. 8. ed. São Paulo: Pearson Addison-Wesley, 2007.

VICENTE, Kim. Homens e máquinas: como a tecnologia pode revolucionar a vida cotidiana. Rio de Janeiro: Ediouro, 2005.

Submetido para avaliação em 16 de setembro de 2011.

Aprovado para publicação em 31 de janeiro de 2014.

Nilton Manoel Lacerda Adão: Professor no SENAI (Serviço Nacional de Aprendizagem Industrial) Centro de Tecnologia em Eletrometalmecânica - Florianópolis - SC - Brasil.

E-mail: niltonadao@hotmail.com

Alexandre Altair de Melo: Professor do Centro de Tecnologia em Automação e Informática e analista de sistemas - TOTVS S.A. - Florianópolis - SC - Brasil. E-mail: alexandre.melo@sc.senai.br

Dieison Grumovski: Professor no SENAI (Serviço Nacional de Aprendizagem Industrial) - Joinville - SC - Brasil. E-mail: dieison.grumovski@sc.senai.br 